\title{
Bias Selective Operation of Sb-Based Two-Color Photodetectors
}

\author{
M.N. Abedin ${ }^{1}$, T.F. Refaat ${ }^{2}$, I.B. Bhat ${ }^{3}$, Y. Xiao ${ }^{3}$, and D.G. Johnson ${ }^{1}$ \\ ${ }^{1}$ NASA Langley Research Center, Hampton, VA \\ ${ }^{2}$ Old Dominion University, Norfolk, VA \\ ${ }^{3}$ Rensselaer Polytechnic Institute, Troy, NY
}

\begin{abstract}
Multicolor detectors have a strong potential to replace conventional single-color detectors in application dealing with the simultaneous detection of more than one wavelength. This will lead to the reduction of heavy and complex optical components now required for spectral discrimination for multi-wavelengths applications. This multicolor technology is simpler, lighter, compact and cheaper with respect to the single-color ones.

In this paper, Sb-based two-color detectors fabrication and characterization are presented. The color separation is achieved by fabricating dual band pn junction on a GaSb substrate. The first band consists of an InGaAsSb pn junction for long wavelength detection, while the second band consists of a GaSb pn junction for shorter wavelength detection. Three metal contacts were deposited to access the individual junctions. Surface morphology of multi-layer thin films and also device characteristics of quasi-dual band photodetector were characterized using standard optical microscope and electro-optic techniques respectively. Dark current measurements illustrated the diode behavior of both lattice-matched detector bands. Spectral response measurements indicated either independent operation of both detectors simultaneously, or selective operation of one detector, by the polarity of the bias voltage, while serially accessing both devices.
\end{abstract}

Keywords: IR Detectors, Two-color detectors, InGaAsSb, GaSb, Bias selective operation.

\section{INTRODUCTION}

A strong interest exists in two-color and multicolor infrared detectors for many applications in military, NASA, and industry. One of the simplest multi-color combinations is the stacked arrangement in which the shorter wavelength detector is placed optically ahead of the longer wavelength detector. The first detector absorbs only the shorter wavelength radiation and transmits the longer wavelength radiation to the second, third detector and so on. Another method to detect multi-wavelength simultaneously is to use classical optical components such as lenses, prisms and gratings to separate out the wavelength before it hits the detectors. However, the most common trend today is to have two-dimensional mosaic arrays of IR detectors that are electronically multiplexed. These are referred to as infrared focal plane arrays (IRFPAs) useful in imaging applications. For this, use of classical optical components is complicated, and use of photovoltaic detectors in the same image area is preferable.

In early 70s, a two-color infrared detector has been demonstrated in HgCdTe through stacked arrangement technique ${ }^{1}$. Two-color HgCdTe FPAs have been used in defense applications to allow simultaneous detection with high quantum efficiency at two bands on the same FPA ${ }^{2}$. In addition, two-color FPAs based on quantum well infrared photodetectors (QWIPs) have been reported by JPL 
group $^{3-4}$. Four-color FPAs have been demonstrated in the 3-to-15 $\mu \mathrm{m}$ wavelength region in the QWIPS technology ${ }^{5}$. But, there is no report on multi-color FPA technology in the spectral range of near UV to long wave, a region that has important potential benefits to NASA Earth Science Enterprise. Therefore, NASA Langley Research Center (LaRC), in partnership with Rensselaer Polytechnic Institute (RPI) and Jet Propulsion Laboratory (JPL), proposed to develop a multi-color detector in the UV to far infrared wavength range by using wafer-bonding technology ${ }^{6-8}$ within Breakthrough Atmospheric Remote Sensing under NASA enabling concepts and technology (ECT) development program.

Recently, RPI fabricated photovoltaic IR detector suitable at two different wavelengths using Sbbased material systems ${ }^{9-13}$, which is the part of the multi-color detector development effort. Due to the wide range of applications in the short wave-to-mid wave infrared (SWIR to MWIR) wavelength range, there is interest in Sb-based infrared photodetectors. GaSb substrate is an attractive choice since the lattice parameters of various GaSb based quaternary III-V compound semiconductors can be tailored to match that of GaSb. Their band gaps cover a wide spectral range from $\sim 0.3$ to 1.58 $\mathrm{eV}$, or a wavelength range from near infrared $(0.8 \mu \mathrm{m})$ to mid-infrared $(4.3 \mu \mathrm{m})$. Numerous optoelectronic device papers have been published in the $1.7-4.4 \mu \mathrm{m}$ wavelength range using Sbbased material system ${ }^{14-20}$, and particularly photodetectors fabricated from quaternary alloy $\mathrm{Ga}_{\mathrm{x}} \mathrm{In}_{1}$ ${ }_{x} \mathrm{As}_{\mathrm{y}} \mathrm{Sb}_{1-\mathrm{y}}$ can provide room temperature operation near the mid-infrared wavelength range ${ }^{14,21}$.

Growth of GaSb based quaternary III-V compound semiconductors using Metal-organic Chemical Vapor Deposition (MOCVD) have been reported in the literature ${ }^{22-25}$. Complex device structures of InAsSb/GaInAsSb/AlGaAsSb/GaSb heterostructures for wafer-bonded thermophotovoltaic devices and. of multi-stage mid-infrared InAsSb emitters have been reported ${ }^{26-}$ 27. Another type of device such as multi-color photodetector, which involves complex heterostructures, is proposed here. Though, GaSb-based multi-color detectors are more practical compared to HgCdTe technology due to the advancement in III-V technology. In addition, the simple stacked structure, where the short wavelength detector is placed optically ahead of the long wavelength one in GaSb is more practical, avoiding complex superlattice layers. This practical approach for developing two-color InGaAsSb/GaSb photodetectors on GaSb substrate has not been investigated much, probably due to the growth difficulty of InGaAsSb on GaSb substrate.

Sb-based materials growth, quasi-dual band detector fabrication and characterization are reported in this article. Dark current with applied bias voltages and spectral response measurements with selectively biased voltage as a function of incident wavelengths are demonstrated.

\section{Sb-BASED MULTILAYER GROWTH AND DEVICE FABRICATION}

\section{A. Multilayer growth}

The growth of multilayer thin films was carried out using an in-house built horizontal MOCVD equipment ${ }^{28}$. The carrier gas is ultra-pure hydrogen $\left(\mathrm{H}_{2}\right)$ at a flow rate of $4500 \mathrm{sccm}$, and the growth pressure is maintained at 100 Torr with growth temperature set to $600{ }^{0} \mathrm{C}$. Organometallic sources include trimethylindium (maintained at room temperature), trimethylgallium (at $\sim-6{ }^{0} \mathrm{C}$ ), tertiarybutylarsine (at $\sim-10{ }^{0} \mathrm{C}$ ), and trimethylantimony (at $\sim 4{ }^{0} \mathrm{C}$ ), and the $\mathrm{n}$ - and p-dopant sources are diethyltelluride (DeTe, at room temperature) and silane diluted in ultra-pure $\mathrm{H}_{2}(100 \mathrm{ppm}$ ), respectively. The growth rate is approximately 3.8-4.0 $\mu \mathrm{m} / \mathrm{hr}$.

Multilayer structures were grown from top to bottom as n-InGaAsSb/p-InGaAsSb/p-GaSb on pGaSb substrate (n-on-p InGaAsSb photodiode), and $\mathrm{p}^{+}-\mathrm{InGaAsSb}$ (or $\mathrm{p}^{+}-\mathrm{GaSb}$ )/p-InGaAsSb/nInGaAsSb/n-GaSb on $n-G a S b$ substrate (p-on-n InGaAsSb photodiode), respectively. The GaSb 
layer immediately above the substrate is a very thin buffer layer grown on GaSb substrate. The p-onn photodiodes were grown on n-type GaSb substrates with two orientations, (100) $2^{0} \rightarrow(110)$ and $(100)+6^{0} \rightarrow(111) B$. The as-grown multilayer films are characterized with optical microscope, atomic force microscope (AFM), X-ray, and scanning electron microprobe. With optimized growth parameters, high quality multilayer films could be repeatedly grown. The typical alloy concentration for InGaAsSb is $\mathrm{In}_{0.13} \mathrm{Ga}_{0.87} \mathrm{As}_{0.11} \mathrm{Sb}_{0.89}$ as determined by $\mathrm{X}$-ray diffraction measurement and transmission electron microprobe analyses. In Figures 1, optical microscope image is shown for the surface morphology of the multilayer thin films of p-on-n InGaAsSb photodiodes. The cross-hatch pattern of the surface morphology is partly alleviated for the p-on-n InGaAsSb photodiode, when the lattice of InGaAsSb is tailored to be close to that of GaSb substrate.

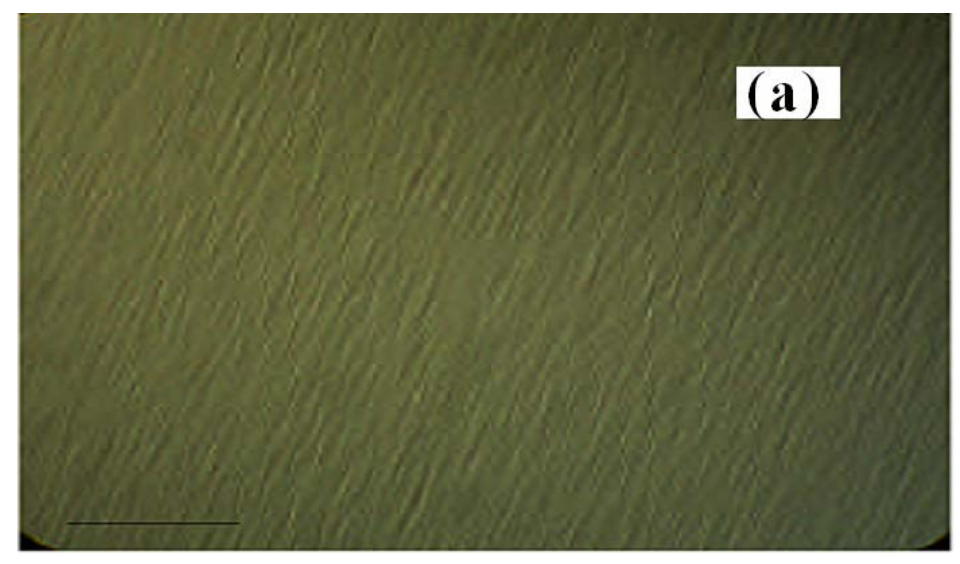

Figure 1. Microscope image for the surface morphology of multi-layer thin films of p-on-n InGaAsSb $\left[\mathrm{P}^{+}\right.$-InGaAsSb/p-InGaAsSb/n-InGaAsSb/n-GaSb thin buffer] photodiodes ${ }^{12}$.

\section{B. Device fabrication}

The selected samples were degreased with hot xylene followed by acetone and methanol rinse (XAM cleaning) at the beginning. Mesas were patterned by using the standard photolithographic technique, and the diode structures were generated with wet chemical etching by using a solution of $\mathrm{HCl}: \mathrm{H}_{2} \mathrm{O}_{2}: \mathrm{NaK}$ tartrate (33ml:9ml:12g in 0.5 liter of solution) ${ }^{29}$. For quasi-dual band photodetectors, two mesa steps were etched to form both the upper and lower photodiodes. The first mesa was etched somewhat near the interface between the GaSb and InGaAsSb layers. Spinned photoresist then covered the first-time etched mesa step whereas the lower photodiode mesa structure was etched down to the substrate. Immediately before loading the samples into the e-beam evaporator for metallization, the samples were immersed in hydrochloric acid $(\mathrm{HCl})$ to remove the native oxide layer. For the smallest quasi-dual band photodetector, the nominal diameter value is set to $225 \mu \mathrm{m}$ in order to enhance the fabrication yield. Particularly for the quasi-dual band photodetectors, the front metallization was done with evaporation of $\mathrm{Pd} / \mathrm{Ge} / \mathrm{Au} / \mathrm{Ti} / \mathrm{Au}$ on both $\mathrm{p}$ - and n-type surfaces/interfaces. The schematic device structure for a quasi-dual band photodetector and the fabricated device image are shown in Figure 2(a) and (b) respectively. 

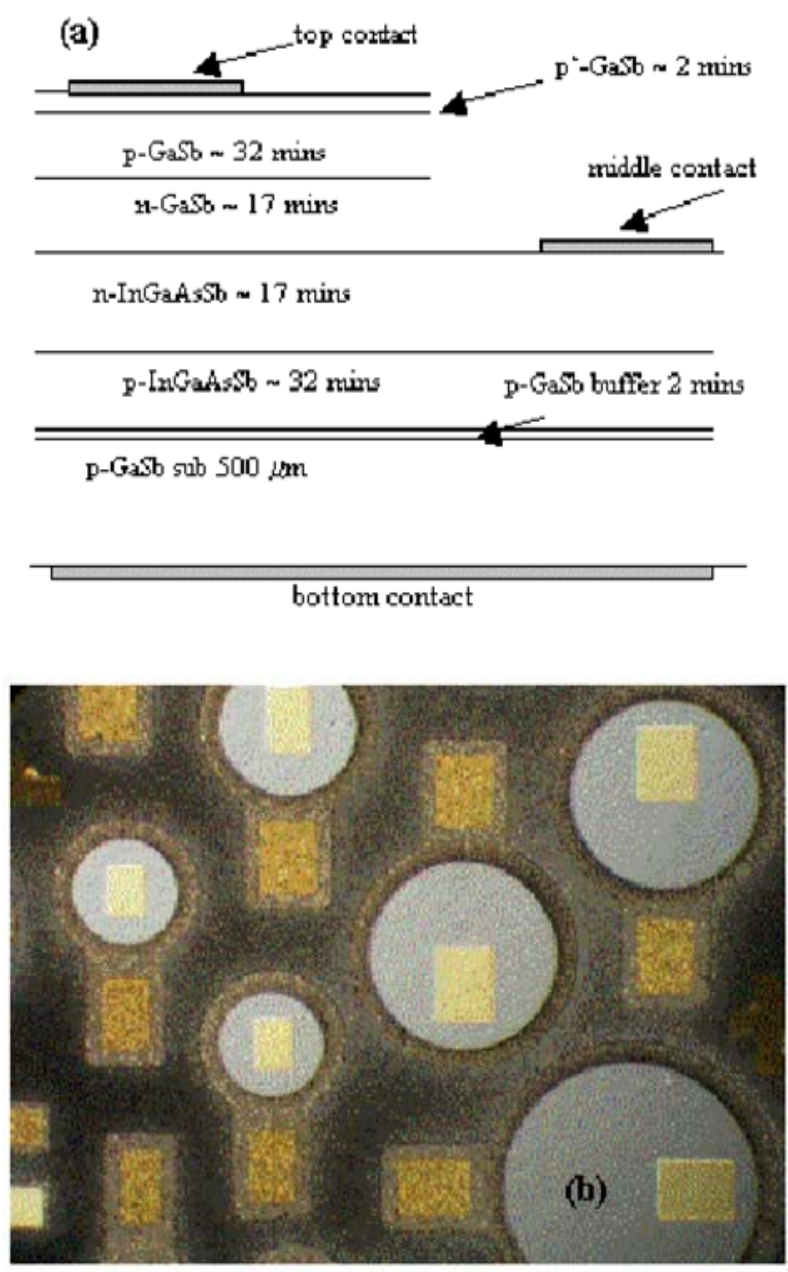

Figure 2. (a) Schematic device structure for a quasi-dual band photodetector and (b) microscope image for the fabricated quasi-dual band (n/p/p/n-on-n) photodetectors ${ }^{12}$.

\section{RESULTS AND DISCUSSIONS}

We will describe dark current and spectral measurement results together with discussion on quasi-dual band photodiodes.

\section{Dual band photodetectors}

Dual band photodetectors have been fabricated on selected multilayer n/p/p/n-on-n and p/n/n/pon-p thin films. Whereas good ohmic contact is hard to achieve on the top n-GaSb surface for the $\mathrm{n} / \mathrm{p} / \mathrm{p} / \mathrm{n}$-on-n structure, the fabricated $\mathrm{p} / \mathrm{n} / \mathrm{n} / \mathrm{p}$-on-p quasi-dual band photodetector demonstrates diode behavior for both the upper and lower photodiodes with various device sizes. The dark I-V curves for one quasi-dual band photodetector are shown in Figure 3. The dark current measurements were performed at $23.5^{\circ} \mathrm{C}$ and higher dark current was obtained from top device as compared to bottom device. The contact still needs improvement for the upper photodiode, and the lower photodiode 
works well. The upper and lower diameters of the photodiodes are $775 \mu \mathrm{m}$ and $900 \mu \mathrm{m}$ respectively. Both of the devices show diodes behavior.

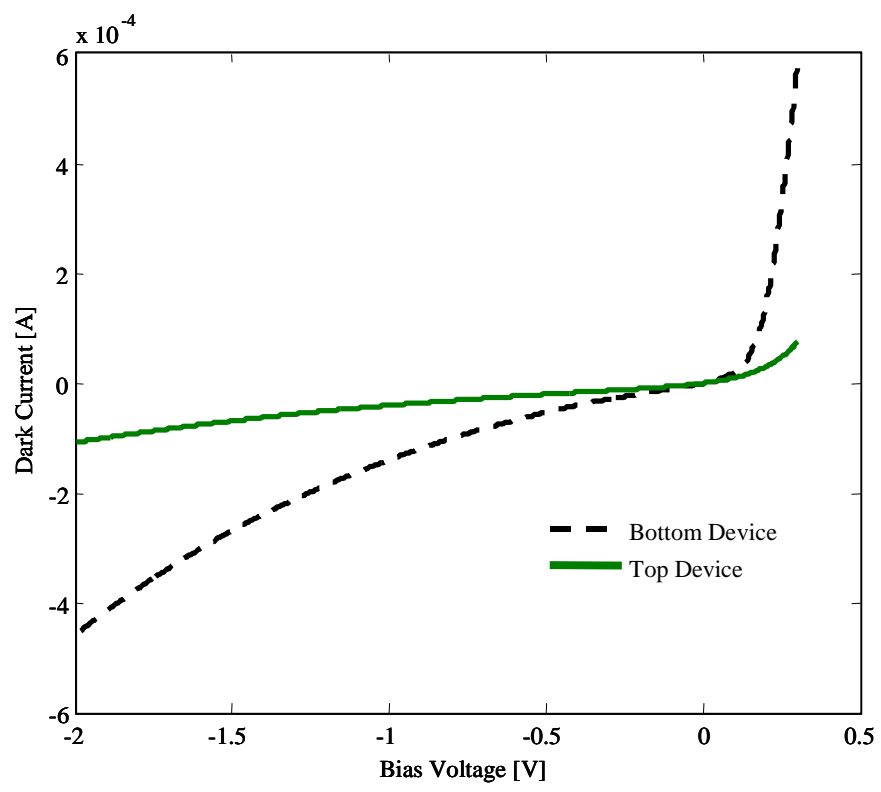

Figure 3. I-V curves for quasi-dual band photodetector.

Responsivity of the dual-band detector is obtained in the 1.0- to 1.7- $\mu \mathrm{m}$ and 1.0- to 2.1- $\mu \mathrm{m}$ by using a PbS calibrated detector. The output signal of the photodetector is acquired and then compared with the calibrated $\mathrm{PbS}$ detector. The spectral responsivity, $R_{T}(\lambda)$ (in $\mathrm{A} / \mathrm{W}$ ), of the photodetector is determined by using the following equation ${ }^{30}$ :

$$
R_{T}(\lambda)=\frac{V_{T}(\lambda)}{V_{P b S}(\lambda)} \cdot \frac{A_{r}}{g} \cdot R_{P b S}(\lambda)
$$

where $V_{P b S}$ is the PbS photodiode response (in $\mathrm{V}$ ), $V_{T}$ is the dual-band photodetector output (in V), $A_{r}$ is the ratio of the PbS detector area to the dual band photodetector area, $g$ (in V/A) is the preamplifier gain setting, and $R_{P b S}$ (in $\mathrm{V} / \mathrm{W}$ ) is the spectral responsivity of the PbS detector.

Responsivity for selected quasi-dual band photodetectors is also measured and the results are shown in Figure 4. The measurements are conducted at room temperature $\left(23.5^{\circ} \mathrm{C}\right)$ and at zero bias voltage. For both devices as a whole, the photoresponse is collected from the top and the bottom contact terminals. Both the upper and lower photodiodes can sense the photon illumination up to their cutoff wavelengths ( $\sim 1.7 \mu \mathrm{m} \& \sim 1.9 \mu \mathrm{m}$ respectively) limited by their corresponding material bandgaps. In Figure 4, for the upper device, the responsivity beyond the $1.7 \mu \mathrm{m}$ could be attributed to the depth uncertainty when etching the first mesa. The over-etched depth may include part of the n-InGaAsSb layer into the upper diode structure. The material composition for the lower photodiode needs to be tailored to achieve the responsivity beyond $2 \mu \mathrm{m}$.

Spectral response measurements of the upper and lower detectors at different bias voltages are shown in Figure 5. During these measurements, the bottom contact was grounded and a positive voltage was applied at the top contact of the serially connected top and bottom devices, which leads 
to the forward bias of one of the p-n junction. A negative voltage at the top contact leads to the reverse bias to other bottom p-n junction. Selectable detection for quasi-dual band detectors by the polarity of the applied bias voltage was shown in Figure 5. Applying biases across the p-n junction changes the responsivity of the detector. The reverse bias increases the depletion width, which enhances drift of the photogenerated charge carriers and the forward bias decreases the depletion region that results in responsivity changes. This behavior is attributed to simultaneous change in the depletion regions with the bias voltage as discussed for the two devices at the same time ${ }^{10}$.

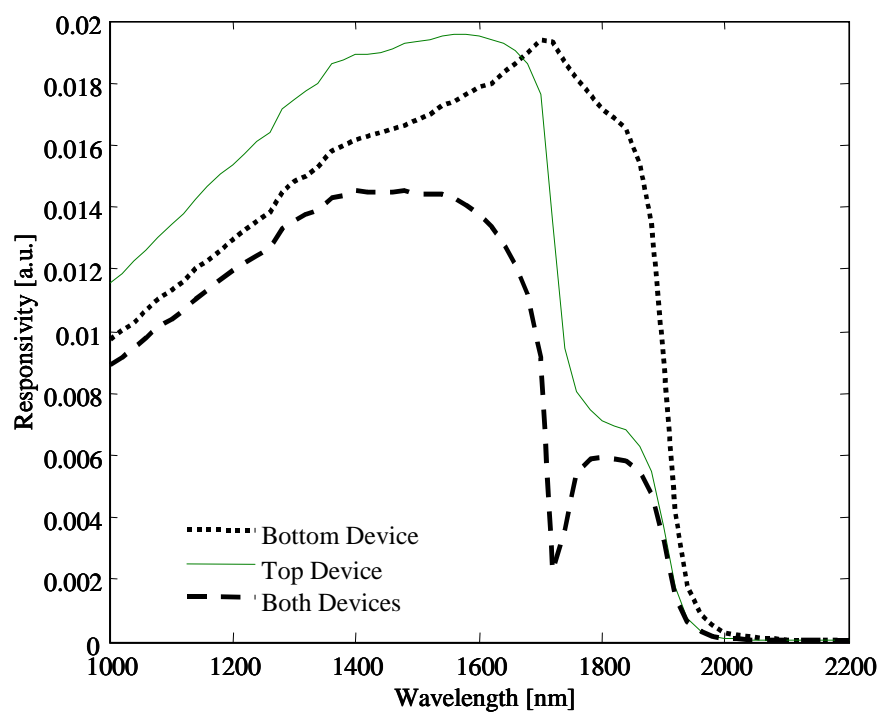

Figure 4. The measured responsivity versus wavelength for one quasi-dual band photodetector. Curves shown are for the upper and the lower device.

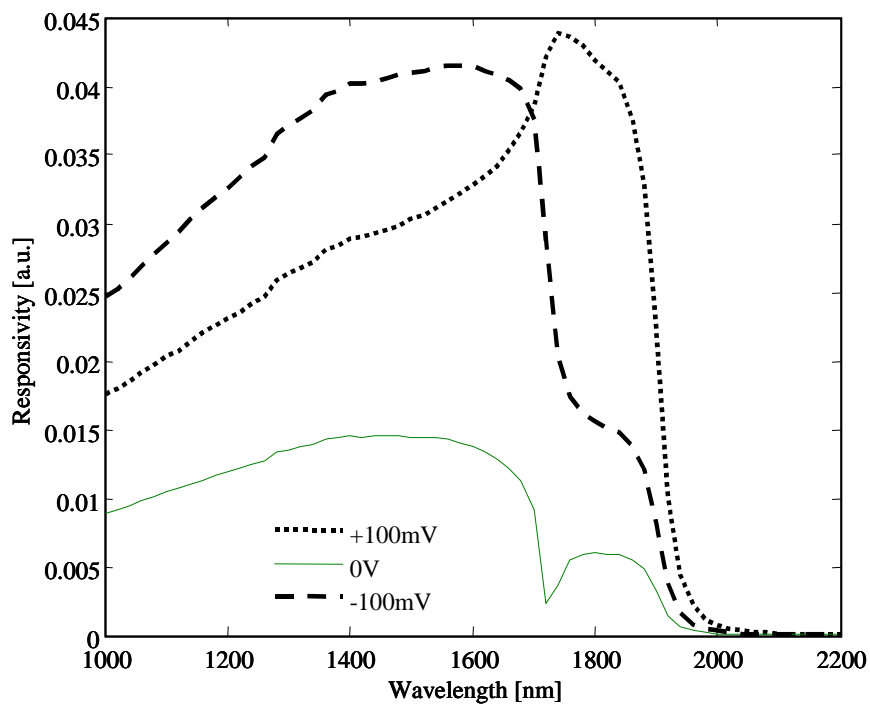

Figure 5. Obtained spectral responses of both detectors by grounding the bottom contact and applying the biases (forward and reverse voltages) at the top contact. 


\section{SUMMARY}

In this work, GaSb based multilayer structures have been grown by using MOVPE. Thin films with good surface morphology can be routinely obtained with optimal growth condition. More complex multilayer structures for quasi-dual band photodetectors (1.7 $\mu \mathrm{m}$ and $\sim 2 \mu \mathrm{m}$ bands) are also investigated, and these quasi-dual band structures include $\mathrm{n}-\mathrm{GaSb} / \mathrm{p}-\mathrm{Gasb} / \mathrm{p}-\mathrm{InGaAsSb} / \mathrm{n}$ -

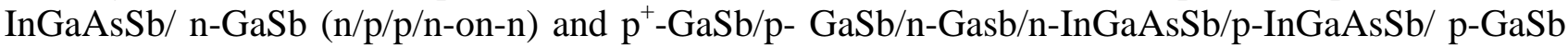
(p/n/n/p-on-p) grown on $n-$ and $\mathrm{p}-\mathrm{GaSb}$ substrates respectively. For $\mathrm{p} / \mathrm{n} / \mathrm{n} / \mathrm{p}-\mathrm{p}-\mathrm{p}$ quasi-dual band photodetectors, both the upper and the lower devices function with typical diode behavior under bias. The responsivity measurements indicate that both the upper and the lower photodiodes can sense illumination up to wavelengths limited by their corresponding material bandgaps. In addition, spectral response measurements indicated either independent operation of both detectors simultaneously, or selective operation of one detector, by the polarity of the bias voltage, while serially accessing both devices. Results show the dual band features for future development of the multi-band detectors.

\section{ACKNOWLEDGEMENT}

This effort is part of the Breakthrough Atmospheric Sensing funded by NASA's Earth Science Technology Office and the Enabling Concepts and Technology Program within NASA's Aerospace Technology Enterprise.

\section{REFERENCES}

1. H. Halpert and B.L.Musicant, "N-Color (Hg, Cd) Te Photodetectors“, Appl. Optics 11 (1972), pp. 2157-2161.

2. M.B. Reine, A. Hairston, P. O’Dette, S.P. Tobin, F.T.J. Smith, B.L. Musicant, P. Mitra, and F.C. Case, "Simultaneous MW/LW dual-band MOCVD HgCdTe 64x64 FPAs", Proc. SPIE 3379 (1998), pp. 200-212.

3. S.D. Gunapala, S.V. Bandara, A. Singh, J.K. Liu, E.M. Luong, J.M. Mumolo, and M.J. McKelvey, "Quantum Well Infrared Photodetectors for Low Background Applications”, Proc. SPIE 3379 (1998), pp. 225-234.

4. S.D. Gunapala, personal communication, Jet Propulsion Laboratory, July 2000.

5. S.V. Bandara, S. D. Gunapala, J. K. Liu, S. B. Rafol, D. Z. Ting, J. M. Mumolo, R.W. Chuang, T.Q. Trinh, J. H. Liu, K. K. Choi, M. Jhabvala, J. M. Fastenau, W. K. Liu "Four-band Quantum Well Infrared Photodetector Array”, Infrared Physics and Technology, Vol. 44, pp. 369-375 (2003).

6. M.N. Abedin, T.F. Refaat, J.M. Zawodny, S.P. Sandford, U.N. Singh, S.V. Bandara, S.D. Gunapala, and I.B. Bhat, "Multicolor Focal Plane Array Detector: A Review", Infrared Spaceborne Remote Sensing XI, SPIE Vol. 5152, pp. 279 - 288 (2003).

7. M.N. Abedin, T.F. Refaat, Y. Xiao, I.B. Bhat, S.V. Bandara, and S.D. Gunapala, "Progress of multicolor single detector to detector array development for remote sensing", Infrared Spaceborne Remote Sensing XII, SPIE Vol. 5543, pp. 239 - 247 (2004).

8. M.N. Abedin, T.F. Refaat, Y. Xiao, and I. Bhat "Characterization of dual-band infrared detectors for application to remote sensing”, Infrared Spaceborne Remote Sensing 2005, Proc. SPIE Vol. 5883, pp. 588307-1 - 5883-8 (2005). 
9. I. Bhat, Y. Xiao, V. Bhagwath, P. Dutta, T.F. Refaat, and M.N. Abedin, "GaInAsSb-based infrared detectors in the 2- $\mu \mathrm{m}$ range fabricated from multi-layers grown by Metalorganic vapor Phase epitaxy”, MSS Workshop, Charleston, SC, February 2005.

10. T.F. Refaat, M.N. Abedin, I.B. Bhat, Y. Xiao, "Sb Based Two-Color Photodetector Fabrication and Characterization”, published in Optical Engineering, 2005.11. Y. Xiao..(SPIE 2005)

12. I. Bhat, Y. Xiao, T.F. Refaat, and M.N. Abedin, "Organometallic Vapor Phase Epitaxy of GaSb/InGaAsSb Multilayers and Fabrication of Dual band Infrared Detectors”, presented at the Twelfth US Biennial Workshop on Organometallic Vapor Phase Epiatxy (OMVPE), Montana, July 10-14, 2005.

13. Y. Xiao, I. Bhat, T.F. Refaat, M.N. Abedin, and Q. Shao, "MOCVD growth of GaSb/InGaAsSb multilayers and fabrication of quasi-dual band photodetectors", published at SPIE $50^{\text {th }}$ Annual Meeting, San Diego, CA (2005).

14. A. K. Srivastava, J. C. DeWinter, C. Caneau, M. A. Pollack, and J. L. Zyskind, "High performance GaInAsSb/GaSb p-n photodiodes for the 1.8-2.3 $\mu \mathrm{m}$ wavelength range," Appl. Phys. Lett. 48, pp. 903-904 (1986).

15. Y.-M. Sun, J.-M. Wang, and M.-C. Wu, "Liquid-phase epitaxial growth of GaInAsSb and the properties of AlGaSb/GaSb/GaInAsSb separate absorption and multiplication avalanche photodiodes,” Jpn. J. Appl. Phys., 35, pp. 5246-5249 (1996).

16. O. V. Sulima, M. G. Mauk, Z. A. Shellenbarger, J. A. Cox, J. V. Li, P. E. Sims, S. Datta, and S. B. Rafol, "Uncooled low-voltage AlGaAsSb/InGaAsSb/GaSb avalanche photodiodes," IEEE Proc. - Optoelectron. 151, pp.1-5 (2004).

17. N. M. Kolchanova, A. A. Popov, G. A. Sukach, and A. B. Bogoslovskaya, "Thermal-processes in GaInAsSb-based light-emitting-diode (LED) heterostructures,” Semiconductors, 28, pp. 11371140 (1994).

18. D. Akhmedov, A. G. Deryagin, S. A. Karandashov, V. I. Kuchinskii, V. M. Smirnov, G.S. Sokolovskii, V. I. Vasil'ev, "GaInAsSb/GaSb double heterostructure light emitting diodes," Proc. SPIE, 3316, pp. 162-164 (1998).

19. C. Caneau, J. L. Zyskind, J. W. Sulhoff, T. E. Glover, J. Centanni, C. A. Burrus, A. G. Dentai, and M.A. Pollock, "2.2 $\mu \mathrm{m}$ GaInAsSb/AlGaAsSb injection lasers with low threshold current density,” Appl. Phys. Lett. 51, pp. $764-766$ (1987).

20. T. H. Chiu, J. L. Zyskind, and W. T. Tsang, "Molecular-beam epitaxial-growth of InGaAsSb on (100) GaSb with emission wavelength in the 2 to $2.5 \mu \mathrm{m}$ range,” J. Electron. Mater. 16, pp. 5761 (1987).

21. C. Caneau, A. K. Srivastava, A. G. Dentai, J. L. Zyskind, and M. A. Pollack, "Room temperature GaInAsSb/AlGaAsSb DH injection-lasers at 2.2 m,” Electron. Lett. 21, pp. 815-817 (1985).

22. G. Bougnot, F. Delannoy, A. Foucaran, F. Pascal, F. Roumanille, P. Grosse and J. Bougnot, "Growth of GaInAsSb alloys by MOCVD and characterization of GaInAsSb/GaSb p-n photodiodes,” J. Electrochem. Soc., 135, pp. 1783-1788 (1988).

23. C. A. Wang, H. K. Choi, D. C. Oakley, and G. W. Charache, "Recent progress in GaInAsSb thermophotovoltaics grown by organometallic vapor-phase epitaxy,” J. Crystal Growth, 195, pp. 346-355 (1998).

24. J. G. Cederberg, M. J. Hafich, R. M. Biefeld, and M. Palmisiano, "The preparation of InGa(As)Sb and $\mathrm{Al}(\mathrm{Ga}) \mathrm{AsSb}$ films and diodes on $\mathrm{GaSb}$ for thermophotovoltaic applications using metal-organic chemical vapor deposition,” J. Crystal Growth, 248, pp. 289-295 (2003). 
25. C. W. Hitchcock, R. J. Gutmann, H. Ehsani, I. B. Bhat, C. A. Wang, M. J. Freeman, and G. W. Charache, "Tenary and quaternary antimonide devices for thermophotovoltaic applications," $J$. Crystal Growth, 195, pp. 363-372 (1998).

26. C. A. Wang, D. A. Shiau, and D. R. Calawa, "Growth and characterization of InAsSb/GaInAsSb/AlGaAsSb/GaSb heterostructures for wafer-bonded thermophotovoltaic devices,” J. Crystal Growth, 261, pp. 372-378 (2004).

27. R. M. Biefeld, A. A. Allerman, S. R. Kurtz, and K. C. Baucom, "Progress in the growth of midinfrared InAsSb emitters by metal-organic chemical vapor deposition,” J. Crystal Growth, 195, pp. 356-362 (1998).

28. H. Ehsani, I. Bhat, C. Hitchcock, R. J. Gutmann, G. Charache, and M. Freeman, "p-type and ntype doping in GaSb and $\mathrm{Ga}_{0.8} \mathrm{In}_{0.2}$ Sb layers grown by metalorganic vapor phase epitaxy", $J$. Crystal Growth, 195, pp. 385-390 (1998).

29. C. W. Hitchcock, R. J. Gutmann, H. Ehsani, I. B. Bhat, C. A. Wang, M. J. Freeman, and G. W. Charache, "Tenary and quaternary antimonide devices for thermophotovoltaic applications," $J$. Crystal Growth, 195, pp. 363-372 (1998).

30. M.N. Abedin, T.F. Refaat, O.V. Sulima, and U.N. Singh, “AlGaAsSb/InGaAsSb heterojunction phototransistors with high optical gain and wide dynamic range”, IEEE Trans. Electron Devices 51(12), 2013-2018 (2004). 